\title{
O PAPEL DO DOCUMENTO ORIGINAL NOS ANAIS DO SNHM DE 1999 A 2015: ESTUDOS PRELIMINARES
}

\section{THE ROLE OF THE ORIGINAL SOURCE IN THE ANNALS OF THE SNHM FROM 1999 TO 2015: PRELIMINARY STUDIES}

\author{
Isabelle Coelho da Silva1 \\ Instituto Federal de Educação Ciência e Tecnologia do Ceará(IFCE)/Programa de \\ Pós-Graduação em Ensino de Ciências e Matemática (PGECM)
}

Ana Carolina Costa Pereira ${ }^{2}$

Universidade Estadual do Ceará (UECE)

\begin{abstract}
Resumo
As recentes publicações acadêmicas e os eventos na área da Educação Matemática nos fazem perceber o crescente número de pesquisas envolvendo o uso da história da matemática em sala de aula. Dentre esses estudos, também notamos que a articulação de fontes históricas, mais especificamente, os documentos originais, para o ensino de matemática tem sido bastante explorada. Esses documentos podem ser qualquer material histórico original presente na sociedade que está sendo estuda, tais como livros, instrumentos, artefatos, certidões, entre vários outros. Nesse sentido, nos questionamos como esse material está sendo utilizado em pesquisas que visam articular história da matemática e ensino, pois é importante investigar se isto está realmente acontecendo e beneficiando a educação matemática. Portanto, nesse artigo, objetiva-se apresentar um recorte de uma pesquisa de mestrado, cujo enfoque é fazer uma primeira análise dos trabalhos completos publicados nos anais do Seminário Nacional de História da Matemática (SNHM) de 1999 a 2015 que utilizam fontes históricas, em particular, documentos originais. Para isso, utilizaremos uma pesquisa bibliográfica e um estudo documental para analisar os dados coletados a partir das publicações que abordam esse uso em um dos principais eventos da área, o SNHM. Esse material mostra que a maioria desses textos contém alguma fonte histórica e, pelo menos metade, são classificados como documentos originais, ou seja, é possível perceber sua importância em pesquisas envolvendo a história da matemática. Assim, espera-se categorizar as formas de utilização das fontes históricas nessas pesquisas, além de investigar as bases teóricas utilizadas para fundamentar esse uso para o ensino.
\end{abstract}

Palavras-chave: História da matemática; Documento original; Anais do SNHM; Articulação entre história da matemática e ensino.

\begin{abstract}
The recent academic publications and the events in the area of mathematics education make us realize the growing number of research involving the use of the history of mathematics in the classroom. Among these studies, we also note that the articulation of historical sources, more specifically, the original sources, for the teaching of

\footnotetext{
${ }^{1}$ isabellecoelhods@gmail.com

2 carolina.pereira@uece.br
} 
mathematics has been quite explored. These documents may be any original historical material present in the society that is being studied, such as books, instruments, artifacts, certificates, among many others. In this way, we have questioned how this material is being used in research that aims to articulate the history of mathematics and teaching, because it is important to investigate whether this is actually happening and benefiting mathematics education. Therefore, this article aims to present a part of a master's research, whose focus is to make a first analysis of the complete works published in the annals of the National Seminar of History of Mathematics (SNHM) from 1999 to 2015 that use historical sources, in particular, original sources. To do this, we will use a bibliographical research and a documentary study to analyze the data collected from the publications that address this use in one of the main events of the area, the SNHM. This material shows that most of these texts contain some historical source and at least half of them are classified as original sources, that is, it is possible to notice their importance in research involving the history of mathematics. Thus, it is expected to categorize the ways of using the historical sources in these studies, as well as to investigate the theoretical bases used to justify this use for the teaching.

Keywords: History of mathematics; Original source; Annals of the SNHM; Articulation between history of mathematics and teaching.

\section{Introdução}

Para pesquisadores na área de ensino de matemática é notável o crescente número de publicações abordando uma possível articulação entre história da matemática e ensino. Por exemplo, os estudos de Fauvel e Maanen (2000), Furinghetti (2008), Miguel e Miorin (2004) e Araman e Batista (2017) mostram o quanto esse campo de pesquisa tem ganhado espaço e credibilidade entre os estudiosos da área.

O uso da História da Matemática para o ensino propõe utilizar a história de determinados conteúdos matemáticos para ensiná-los. Desta forma, pode-se explorar como o conteúdo foi descoberto e desenvolvido, qual a motivação inicial do matemático para estudar sobre o tema, como determinada formula foi provada na época de seu descobrimento, dentre outras formas. Miguel e Miorin (2004) defendem o uso da história para o ensino, e apontam os motivos pedagógicos para o seu uso. Estes objetivos levarão os alunos a perceberem

\footnotetext{
1)A matemática como uma criação humana; 2) as razões pelas quais as pessoas fazem Matemática; 3) as necessidades práticas, econômicas e físicas que servem de estímulo ao desenvolvimento das ideias matemáticas; 4) as conexões existentes entre matemática e filosofia, matemática e religião, matemática e lógica, etc.;5) a curiosidade estritamente intelectual que pode levar a generalização e extensão de ideias e teorias; 6) as percepções que os matemáticos têm do próprio objeto da matemática, as quais mudam e se
} 
desenvolvem ao longo do tempo; 7) a natureza da uma estrutura, de uma axiomatização e de uma prova. (MIGUEL; MIORIN, 2004, p. 53)

Dessa forma, percebe-se que utilizar aspectos históricos para discutir ou introduzir um conteúdo vai além de mostrar a sua evolução cronológica, mas também visa entender o papel que o conceito matemático desempenhava em determinadas sociedades e em determinados períodos. Com esse propósito, é necessário investigar todo contexto social que possa ter influenciado o uso e o desenvolvimento da matemática, incluindo os aspectos epistemológicos, historiográficos e contextuais, que são as três esferas de análise para uma pesquisa em história da ciência discutidas por Alfonso-Goldfarb (2008).

Nesse sentido, o uso da História da Matemática requer um entendimento sobre como inserir esta tendência na sala de aula. Para isto, Baroni, Teixeira e Nobre (2004) relataram cinco formas de utilizar este recurso: o desenvolvimento de projetos inspirados pela história, os aspectos culturais da Matemática numa perspectiva histórica, o tratamento detalhado de exemplos particulares, o aperfeiçoamento do conhecimento matemático por meio da História e o uso de fontes originais.

Percebe-se que o uso de fontes históricas, em particular, os documentos originais têm sido cada vez mais abordados em pesquisas que envolvem história da matemática. Saito e Dias (2013), Pereira et al (2016), Silva (2013), entre outros, trazem essa discussão e algumas propostas para sua inserção no ensino.

Fontes históricas são materiais provenientes de uma determinada sociedade que visam comprovar as histórias e descobertas que ocorreram nesta comunidade no período delimitado. Estes podem ser documentos escritos ou artefatos deixados por civilizações antigas. Segundo Xavier (2011, p. 1100), as fontes históricas seriam "todos os tipos de vestígios inscritos no passado, como livros de receita, fotografias, cinema, música, enfim, uma série de elementos que auxiliariam o historiador na busca de compreender os homens do passado e como estes se estabeleceram".

Para o ensino de matemática, a utilização de fontes históricas é indispensável, pois as sociedades antigas fizeram vários estudos e descobertas matemáticas e, portanto, existe uma necessidade da investigação de documentos que comprovem estes fatos e mostrem como e por que estes estudos foram feitos.

Neste sentido, devido ao crescente número de publicações envolvendo o uso de fontes, mais especificamente, os documentos originais, para o Ensino de matemática, 
sentimos a necessidade de examinar esses estudos. Portanto, neste trabalho objetiva-se apresentar um recorte de uma pesquisa de mestrado, cujo enfoque é fazer uma primeira análise dos trabalhos completos publicados nos anais do SNHM de 1999 à 2015 que utilizam fontes históricas, em particular, documentos originais.

\section{O uso de originais para articular história e ensino de matemática}

Não é fácil estudar uma fonte histórica, principalmente, os documentos originais, portanto pensar em uma articulação para o ensino de matemática é uma tarefa ainda mais árdua. Dessa forma, o pesquisador precisaria de tempo e dedicação, além um conhecimento profundo sobre a fonte, a sua linguagem, a sociedade em que está inserida e a matemática que pode ser mobilizada nela.

Nesse sentido, Bacellar (2006) cita alguns aspectos que a pesquisa nessa área precisa seguir para trabalhar com documentos originais, dentre eles estão: conhecer a sua origem; contextualizá-lo e entender o texto no contexto de sua época; discutir os critérios possivelmente adotados por quem a produziu; investigar a qualidade das informações coletadas; comparar e examinar as informações coletadas, justapondo documentos, relacionando texto e contexto, estabelecendo constantes e identificando mudanças e permanências. Posto isso, pode-se validar a utilização das fontes para as pesquisas, além de possibilitar o seu aproveitamento para o ensino.

Contudo, mesmo com as dificuldades em utilizar os documentos originais para o ensino, Jahnke et al (2000) acreditam que, embora seja uma iniciativa ambiciosa, esse é um recurso muito gratificante que trata o conhecimento matemático com um aprofundamento substancial. Assim, pode-se entender alguns preceitos da matemática de forma contextualizada, ou seja, inserida no ambiente em que foi inicialmente desenvolvida ou estudada. Isso faz com que o aluno possa ver as dificuldades encontradas pelos estudiosos do passado, possivelmente identificando seus próprios anseios.

Nessa perspectiva, para pensar em uma possível articulação entre história da matemática e ensino a partir de documentos históricos originais, o pesquisador precisa ter definido a perspectiva historiográfica que irá utilizar: tradicional ou atualizada. A narrativa atualizada se comprometeria a investigar o processo de construção do conhecimento, não apenas tratando-o como uma sequência de fatos estudados com base 
no conhecimento matemático do presente, o que é feito na historiografia tradicional. (SAITO, 2015).

Assim, com a perspectiva historiográfica atualizada, o estudo de um documento original seria feito no ambiente em que está inserido, observando as relações matemáticas e contextuais (tais como, sociais, políticas, religiosas, etc.) a fim de mapear os conhecimentos que o autor mobilizou, sem empregar conceitos estudados posteriormente (SAITO, 2015). Portanto, a partir desses preceitos, pretendemos estudar as pesquisas feitas com a articulação de documentos originais para o ensino, objetivando investigar essas utilizações como uma forma metodológica.

\section{Metodologia}

Este projeto de pesquisa terá como base a pesquisa bibliográfica que, segundo Matos e Vieira (2001), será necessária quando começamos a nos interessar por algum tema, em que "vamos nos familiarizando com literaturas a esse respeito. Assim, estabelecemos uma sintonia entre a nossa proposta de reflexão e o tratamento já dispensado ao assunto por outros pesquisadores".

Em seguida, será adentrado no estudo documental para pesquisar as publicações da área. A partir da definição de fonte histórica dada por Xavier (2011), que foi adotada para este estudo, a pesquisa documental se adequa ao seu propósito, pois uma de suas características "é que a fonte de coleta de dados está restrita a documentos, escritos ou não, constituindo o que se denomina de fontes primárias. Estas podem ser feitas no momento em que o fato ou fenômeno ocorre, ou depois" (MARCONI; LAKATOS, 2003).

Desse modo, serão investigados os anais de um dos principais eventos para pesquisadores deste campo de conhecimento, o Seminário Nacional de História da Matemática. A sua primeira edição ocorreu em 1995, contudo, para este trabalho, a coleta englobará o período de 1999, ano em que foi criada a Sociedade Brasileira de História da Matemática, à 2015. A última edição deste evento ocorreu em 2017, mas como seus anais ainda não foram publicados, estes trabalhos não foram coletados.

A pesquisa nesse evento é necessária devido à sua abrangência entre os estudiosos, pois ele reúne alguns dos principais indivíduos envolvidos com a história da matemática, podendo servir de olhar inicial para visualizar as pesquisas feitas na área. 
Assim, a seleção dos trabalhos foi feita a partir da leitura dos seus títulos, resumos, objetivo e referências, procurando identificar aqueles que envolvessem alguma fonte histórica, em particular, um documento original.

\section{Apresentação dos dados iniciais}

O Seminário Nacional de História da Matemática (SNHM) está na sua XII edição, mas, conforme já mencionado, o recorte deste artigo trabalhará somente os anais dos eventos ocorridos entre 1999 à 2015, ou seja, foram abordados os arquivos da III à XI edições.

Os anais III à VIII utilizados foram impressos e quase todas as publicações foram coletadas, com exceção das escritas em língua espanhola, devido ao pouco número de textos presentes. Isso acontecia em razão de, até o VIII SNHM, apenas alguns trabalhos apresentados durante o evento eram selecionados para publicitação.

Os arquivos das edições IX, X e XI estão disponíveis para consulta online, contudo apenas as publicações classificadas como comunicação oral foram utilizadas, pois os pôsteres não apresentariam material suficiente para uma futura análise completa.

O gráfico 01 a seguir mostra, de forma quantitativa, a coleta feita para este artigo, indicando a comparação entre o número de trabalhos coletados e a quantidade que utiliza algum tipo de fonte histórica na publicação, seja fonte primária, secundária, didática, oral, etc. 
Gráfico 01 - Coleta de dados inicial: trabalhos do SNHM que envolvem fontes

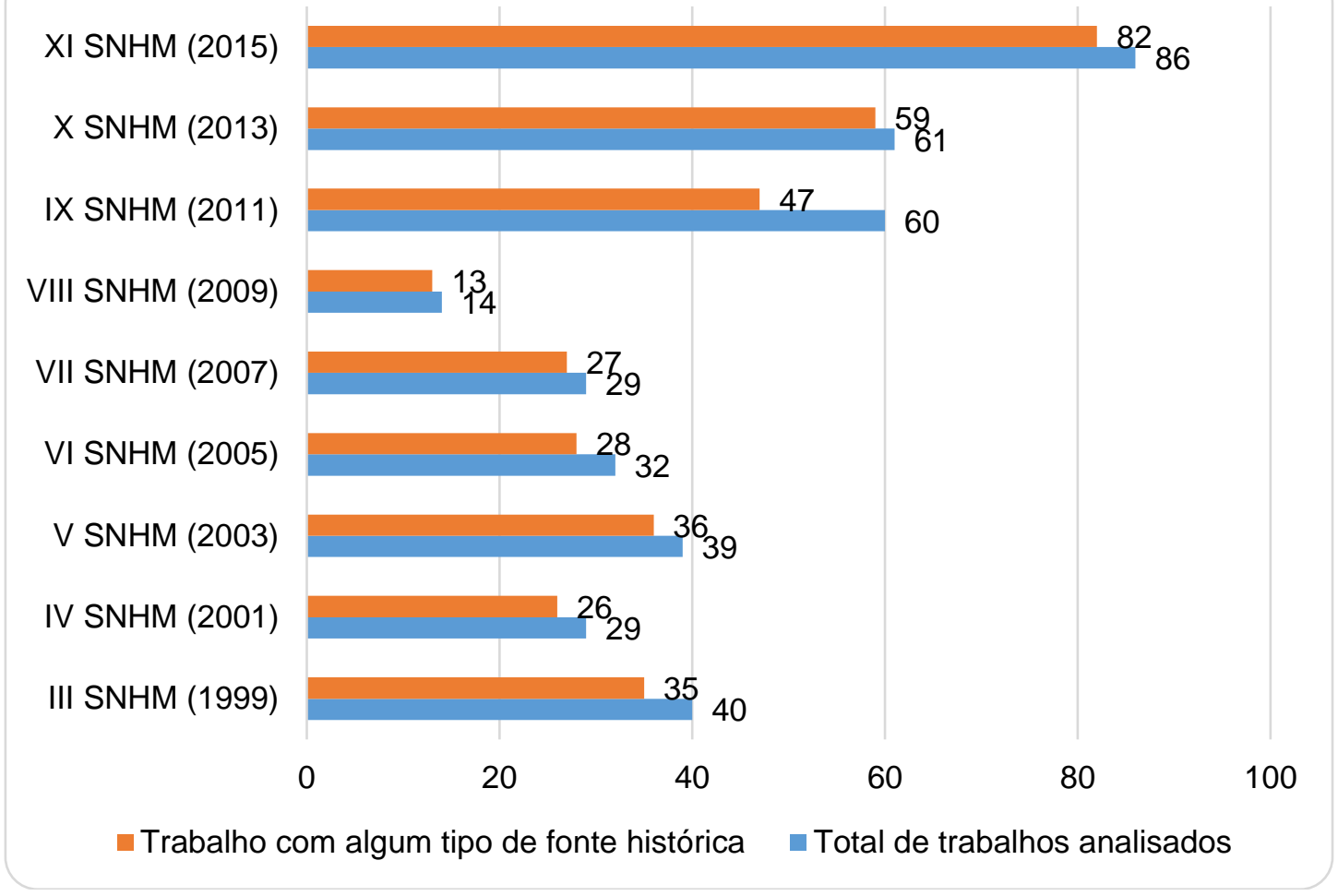

Fonte: Anais do SNHM de 1999 à 2015. Elaborado pelas autoras.

Esses dados nos mostram a grande importância do uso de fontes nos estudos relacionados à história da matemática, pois pelo menos $78 \%$ dos trabalhos publicados em cada edição em questão utilizam de alguma forma uma fonte histórica.

O seguinte gráfico (Gráfico 02) traz, quantitativamente, a coleta feita para este artigo, mostrando uma comparação entre o total de trabalhos analisados e aqueles que envolvem algum documento original, seguindo a definição dada por Xavier (2011). 
Gráfico 02 - Coleta de dados inicial: trabalhos do SNHM que envolvem documentos originais

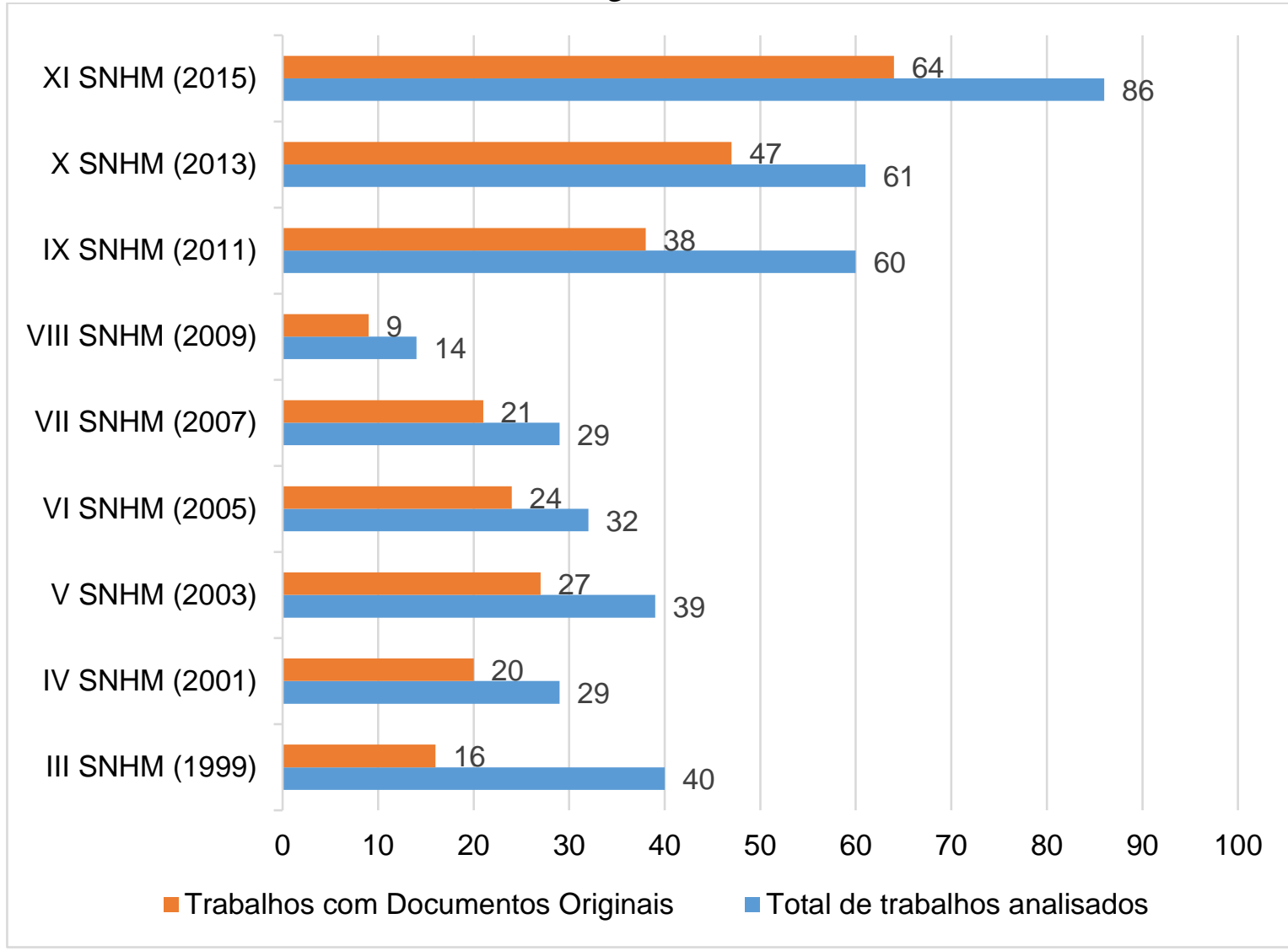

Fonte: Anais do SNHM de 1999 a 2015. Elaborado pelas autoras.

A partir desse gráfico, pode-se ver que a maioria dos anais, com exceção do publicado na III edição do SNHM, possui mais da metade dos trabalhos envolvendo o uso de um documento original, sejam eles textos, artefatos, instrumentos, etc.

Assim, percebe-se a importância das fontes históricas, em particular, os documentos originais, nas pesquisas que envolvem história da matemática em seu âmbito mais geral, sem uma associação ao ensino. Entretanto, nesse primeiro olhar, já é possível visualizar essa relação em alguns dos trabalhos publicados, uma etapa que será realizada posteriormente.

\section{Considerações finais}

Para o professor de matemática da atualidade, não é fácil pensar em diversas técnicas de ensino para melhorar o aprendizado dos alunos, devido ao pouco tempo para realizar planejamentos e à falta de formação continuada para adentrar em pesquisas em áreas como a História da Matemática. Além disso, o estudo de fontes históricas 
demanda tempo e dedicação, o que dificultaria a exploração de uma adequada estratégia de ensino a partir delas.

Dessa forma, para propor uma aula de matemática a partir de uma fonte histórica, é necessário bastante estudo sobre a mesma e a matemática que aborda, além de uma contextualização da sociedade em que ela estava inserida e definição da perspectiva historiográfica que deseja adotar. A partir desses pressupostos, nos perguntamos como as pesquisas que foram feitas no período de 1999 à 2015 estão fazendo essa abordagem e quais os seus pressupostos teóricos.

Nesse primeiro momento, o intuito era trazer uma visão panorâmica sobre o uso de fonte em um evento de grande importância na área, o Seminário Nacional de História da Matemática. A partir dessa coleta, pode-se perceber a importância dessa utilização, seja voltada para o ensino ou não.

Ainda será necessária uma análise mais profunda sobre esses trabalhos, à luz de critérios bem estabelecidos e diversas leituras. Esse será o próximo passo dessa pesquisa, em que estudaremos aqueles trabalhos que propõem articular um documento original para o ensino de matemática, buscando categorizar as formas que os pesquisadores brasileiros estão utilizando-os e investigar a base teóricas utilizada para fundamentar esse uso.

\section{Referências}

ALFONSO-GOLDFARB, A. M. Centenário Simão Mathias: documentos, métodos e identidade da história da ciência. Circunscribere, v. 4, n. 6, p. 5-9, 2008.

ARAMAN, E. M. de O.; BATISTA, I. de L. O Processo de Construção de Abordagens Históricas na Formação Interdisciplinar do Professor de Matemática. Bolema, Rio Claro, v. 31, n. 57, p. 380-407, abr. 2017.

BACELLAR, C. Uso e mau uso dos arquivos. In: PINSKY, Carla Bassanezi et al. Fontes históricas.2. ed. São Paulo: Contexto, 2006. Cap. 2. p. 23-80.

BARONI, R. L. S. TEIXEIRA, M. V.; NOBRE, S. R. A investigação científica em História da Matemática e suas relações com o programa de pós-graduação em Educação Matemática. In: BICUDO, Maria A. V.; BORBA, Marcelo C. Educação Matemática: pesquisa em movimento. São Paulo: Cortez, 2004. p. 164-185.

FAUVEL, J.; MAANEN, J. V. History in mathematics education: the ICMI study. Dordrecht: Kluwer Academic. 2000. 
FURINGHETTI, F. et al. The role of the history of mathematics in mathematics education. In: INTERNATIONAL CONGRESS ON MATHEMATICAL EDUCATION, 11., 2008, Monterrey. Proceedings. Monterrey, 2008. v. 1, p. 1 - 4. Disponível em: <http://www.mathunion.org/fileadmin/ICMI/files/Digital_Library/ICMEs/TSG_23_Rep ort_BB_FF.pdf >. Acesso em: 11 jul. 2017.

JAHNKE, H. N. et al. The use of original sources in the mathematics classroom. In: FAUVEL, John; MAANEN, Jan van. (eds). History in mathematics education: the ICMI study. Dordrecht: Kluwer, 2000. Cap. 9. p. 291-328

MARCONI, M. de A; LAKATOS, E. M. Fundamentos de metodologia científica. $5^{\text {a }}$ ed. São Paulo: Editora Atlas. 2003.

MATOS, K. S. L.; VIEIRA, S. L. Pesquisa educacional: o prazer de conhecer. Fortaleza: Demócrito Rocha, 2001. 143 p.

MIGUEL, A; MIORIN, M. A. História na Educação Matemática: propostas e desafios. Belo Horizonte: Autêntica, 2004. 200 p.

PEREIRA, A. C. C. et al. Sobre o uso de fontes na disciplina de História da Matemática: Problema 56 do Papiro de Rhind. Revemat: Revista Eletrônica de Educação Matemática, Florianópolis, v. 10, n. 2, p. 243-257, jan. 2016. ISSN 19811322.

SAITO, F. História da matemática e suas (re)construções contextuais. São Paulo: Ed. Livraria da Física/SBHMat, 2015.

SAITO, F.; DIAS, M. da S. Interface entre história da matemática e ensino: uma atividade desenvolvida com base num documento do século XVI. Ciênc. educ. (Bauru), Bauru, v. 19, n. 1, p. 89-111, 2013.

SEMINÁRIO NACIONAL DE HISTÓRIA DA MATEMÁTICA, 10., 2013, Campinas. Anais... Disponível em: 〈https://www.cle.unicamp.br/eprints/index.php/anais-snhm>. Acesso em: 07 jan. 2018.

SEMINÁRIO NACIONAL DE HISTÓRIA DA MATEMÁTICA, 11., 2015, Natal. Anais... Disponível em: <http://www.sbhmat.org/crbst_27.html〉. Acesso em: 07 jan. 2018.

SEMINÁRIO NACIONAL DE HISTÓRIA DA MATEMÁTICA, 3., 1999, Vitória. Anais... Vitória: Edufes, 1999. 658 p.

SEMINÁRIO NACIONAL DE HISTÓRIA DA MATEMÁTICA, 4., 2001, Natal. Anais... Rio Claro: Sbhmat, 2001. 392 p.

SEMINÁRIO NACIONAL DE HISTÓRIA DA MATEMÁTICA, 5., 2003, Rio Claro. Anais... Rio Claro: Sbhmat, 2003. 476 p. 
SEMINÁRIO NACIONAL DE HISTÓRIA DA MATEMÁTICA, 6., 2005, Brasília. Anais... Rio Claro: L.A.S., 2005. 354 p.

SEMINÁRIO NACIONAL DE HISTÓRIA DA MATEMÁTICA, 7., 2007, Guarapuava. Anais... Guarapuava: Unicentro, 2008. 274 p.

SEMINÁRIO NACIONAL DE HISTÓRIA DA MATEMÁTICA, 8., 2009, Belém. Anais... Belém: Sbhmat, 2010. 260 p.

SEMINÁRIO NACIONAL DE HISTÓRIA DA MATEMÁTICA, 9., 2011, Aracaju. Anais... Disponível em: 〈http://www.each.usp.br/ixsnhm/Anaisixsnhm/index.php〉. Acesso em: 07 jan. 2018.

SILVA, A. P. P. do N. A leitura de fontes antigas e a formação de um corpo interdisciplinar de conhecimentos: um exemplo a partir do Almagesto de Ptolomeu. 95 f. Dissertação (Mestrado) - Curso de Mestrado em Ensino de Ciências Naturais e Matemática, Universidade Federal do Rio Grande do Norte, Natal, 2013.

XAVIER, E. da S. O uso das fontes históricas como ferramentas na produção de conhecimento histórico: a canção como mediador. Antíteses, vol. 3, n. 6, jul.-dez. de 2010, pp. 1097-1112. 